\title{
O uso da acepromazina, dexmedetomidina e xilazina na sedação em cães: alterações hematológicas e bioquímicas*
}

\section{Acepromazine, dexmedetomidine and xilazine on the sedation in dogs: hematological and biochemical alterations}

\author{
Adriane Picioli, ${ }^{* *}$ Manuele Virgilio Martini, ${ }^{* * *}$ Antonio Humberto Hamad Minervino, ${ }^{* * * *}$ Luis Gustavo Gosuen Gonçalves Dias, ${ }^{* * * *}$ \\ Ewaldo de Mattos Junior******
}

\begin{abstract}
Resumo
Foram avaliados os efeitos hematológicos e bioquímicos mediante a sedação com acepromazina, dexmedetomidina ou xilazina em cães. Para tanto, os animais foram distribuídos aleatoriamente em 3 tratamentos denominados GA (acepromazina 0,1 mg/ $\mathrm{kg}$ ), GD (dexmedetomidina 0,01 mg/kg) e GX (xilazina $1 \mathrm{mg} / \mathrm{kg}$ ) por via i.m. Os parâmetros verificados foram eritrócitos totais, hematócrito $(\mathrm{Ht})$, hemoglobina $(\mathrm{Hb})$, leucócitos totais, proteínas totais, alanina transferase, aspartato transferase, albumina, fosfatase alcalina, gama-glutamil transferase, creatinina e ureia plasmática. Os dados foram coletados nos momentos T0 a T480, sendo T0 previamente a aplicação dos fármacos e o restante dos momentos 15, 30, 60, 120, 240 e 480 minutos após a aplicação dos agentes. Foi observada redução dos eritrócitos totais em todos os momentos comparados ao basal no GA. Houve redução dos valores da $\mathrm{Hb}$ e do $\mathrm{Ht}$ durante todos os momentos de avaliação comparativamente a T0 no grupo GA e GX. Houve redução significativa dos leucócitos totais nos momentos T30, T60, T120 e T240 no GA comparativamente ao momento T0; já nos grupos GD e GX os valores médios foram inferiores ao T0 nos tempos T30 e T120, respectivamente. Não foram verificadas diferenças estatisticamente significativas nas variáveis bioquímicas analisadas entre os tempos e grupos. Baseado nos resultados encontrados, pode-se concluir que a acepromazina e a xilazina promovem redução dos valores dos eritrócitos totais, Ht e Hb em até 8 horas após a sua aplicação, e que todos os fármacos empregados não induzem a alterações hepáticas fundamentada na avaliação enzimática empregada durante este período de avaliação.
\end{abstract}

Palavras-chave: agonistas alfa 2-adrenérgicos, Canis lupus familiaris, fenotiazínicos.

\begin{abstract}
The aim of this study was to evaluate the hematological and biochemical alterations in dogs submitted to sedation with acepromazine, dexmedetomidina or xylazine. Animals were randomly assigned to one of three treatments groups, as follows: GA (acepromazine 0.1 $\mathrm{mg} / \mathrm{kg}$ ); GD (dexmedetomidine $0.01 \mathrm{mg} / \mathrm{kg}$ ); and 3) GX (xylazine $1 \mathrm{mg} / \mathrm{kg}$ ), injected intramuscularly. The parameters evaluated were total erythrocytes, hematocrit $(\mathrm{Ht})$, hemoglobin $(\mathrm{Hb})$, total leukocyte count, total protein, and concentrations of alanine transferase, aspartate transferase, albumin, alkaline phosphatase, gamma glutamyl transferase, creatinine, and urea. Data were collected prior to administration of the drugs (T0) and at 15, 30,60,120,240, and 480 minutes after administration. The was a reduction in total erythrocyte count at all times compared to baseline (GA group). Values of $\mathrm{Hb}$ and Ht were lower compared to T0 in groups $\mathrm{GA}$ and GX at all time points. In the GA group, there was a reduction in total leukocytes at observation times T30, T60, T120, and T240 compared to T0; in groups GX and GD, mean values of total leukocytes were lower at T30 and T120, respectively, compared to T0. There were no differences in biochemical values between observation times and treatment groups. Results indicate that acepromazine and xylazine promote reduction in total erythrocytes count, $\mathrm{Ht}$, and $\mathrm{Hb}$ within 8 hours of administration, and that all drugs used in this study do not induce liver changes based on enzymatic profiles.
\end{abstract}

Keywords: Alpha-2 agonists, Canis lupus familiaris, phenotiazines.

* Recebido em 16 de dezembro de 2012 e aceito em 4 de março de 2013.

** Acadêmica do curso de graduação em medicina veterinária do Centro Universitário de Maringá, Maringá, PR. Bolsista de iniciação científica do PROBIC/ CESUMAR.

*** Bacharel em química, técnica do laboratório de patologia clínica do hospital veterinário do Centro Universitário de Maringá, Maringá, PR.

**** Prof. Dr. da Universidade do Oeste do Pará, Santarém, PA.

${ }^{\star * \star * \star}$ Prof. Dr. de Radiologia Veterinária e do curso de mestrado em medicina veterinária de pequenos animais da Universidade de Franca, Franca, SP.

${ }^{* * * * * *}$ Prof. Dr. de Anestesiologia Veterinária e do curso de mestrado em medicina veterinária de pequenos animais da Universidade de Franca, Franca, SP. ewaldomattos@hotmail.com 


\section{Introdução}

Durante a realização dos procedimentos cirúrgicos e consequentemente anestésicos, é comum ocorrer intercorrências que podem induzir importantes alterações sobre diferentes órgãos e sistemas.

Há uma grande variação nas perdas sanguíneas durante a cirurgia, sendo isso normalmente determinado pelo tipo ou local onde o procedimento está sendo realizado (Tocci 2010). Sabese que perdas de 15 a $20 \%$ do volume sanguíneo já culminam com o surgimento de importantes alterações hemodinâmicas, demonstrando efeitos deletérios (Lanevschi e Wardrop, 2001; Tocci 2010). Com isso, o emprego de fármacos que levam a sequestro volêmico nos diferentes órgãos ou sistemas podem potencializar tais alterações (Lang et al., 1979).

A maioria dos fármacos utilizados na anestesiologia são biotransformados a nível hepático e na iminente necessidade da associação de substâncias poderão ocorrer interações medicamentosas. Essas, por sua vez, podem gerar sobrecarga hepática e consequentemente redução da eliminação das substâncias, influenciando diretamente nas variáveis hemodinâmicas e no período de recuperação anestésica (Muir, 2007).

Dentre os fármacos utilizados para tranquilização na anestesiologia veterinária destacam-se os fenotiazínicos e os agonistas alfa 2-adrenérgicos (Ambrisko e Hikasa, 2002; Kojima et al., 2002) . A acepromazina é o derivado fenotiazínico mais empregado na medicina veterinária (Cortopassi e Fantoni, 2010), produzindo efeitos tranquilizantes mediante a atuação no sistema nervoso central. É biotransformado no fígado, formando inúmeros metabólitos que são eliminados pelas fezes e urina (Neves, 2003). Seu principal efeito colateral é a hipotensão arterial, que ocorre em função do bloqueio de receptores alfa 1-adrenérgicos periféricos (Farver et al., 1986) podendo assim influenciar diretamente os resultados de hematócrito devido à ocorrência de migração celular para a periferia (Lang et al., 1979).

Dos agonistas alfa 2-adrenérgicos, destacam-se a xilazina e dexmedetomidina. Tais agentes causam sedação dosedependente pela estimulação dos alfa 2 adrenorreceptores no sistema nervoso central (Cortopassi e Fantoni, 2010). A biotransformação destas substâncias também ocorre a nível hepático por meio de enzimas do citocromo P450 (Panzer et al., 2011). Após aplicação destes fármacos, normalmente observase redução do hematócrito ligado diretamente à vasodilatação esplênica (Thurmon et al., 1996).

Importantes alterações hematológicas e bioquímicas já foram descritas em pacientes humanos após o emprego dos fenotiazínicos (Dima, 2009) e em animais com agonistas alfa 2-adrenérgicos (Ambrisko e Hikasa, 2002), porém os dados sobre essas informações a nível agudo são escassas. Desta forma, o presente estudo teve como objetivo verificar os efeitos hematológicos e bioquímicos da acepromazina, dexmedetomidina e xilazina por até 8 horas após sua aplicação, partindo da hipótese de que tais fármacos possam induzir a alterações hematológicas e bioquímicas agudas em cães.

\section{Material e métodos}

O estudo foi aprovado pelo Comitê de Bioética Animal do Centro Universitário de Maringá (COBAC), protocolado sob o número 018/2012 e todas as etapas do estudo foram conduzidas seguindo-se os princípios éticos e boas práticas de experimentação animal determinadas pelo Colégio Brasileiro de Experimentação Animal.

\section{Animais}

Foram utilizados seis cães adultos (quatro fêmeas e dois machos), sem raça definida, castrados, provenientes do canil do hospital veterinário do CESUMAR, com peso médio de 10,4 $\pm 3 \mathrm{~kg}$, e idade de $4 \pm 1,1$ anos, clinicamente hígidos, avaliados por meio de exame físico, avaliação hematológica e bioquímica renal e hepática. Previamente ao estudo, foi estabelecido jejum prévio para sólido de 12 horas e líquido de 6 horas.

\section{Delineamento experimental}

Os cães foram selecionados para receber um dos tratamentos em ordem aleatória por método de sorteio, em estudo do tipo "cruzado", respeitando-se o intervalo mínimo de 7 dias entre os tratamentos. Dessa forma, os animais foram distribuídos em 3 tratamentos designados como grupo GA (acepromazina 0,1 mg/ kg, Acepran 0,2\%, Vetnil, Brasil), GD (dexmedetomidina 0,01 mg/ $\mathrm{kg}$, Dexdomitor 0,5 mg/mL, Pfizer, Inglaterra) e GX (xilazina 1 $\mathrm{mg} / \mathrm{kg}$, Rompum 20 mg/mL; Bayer, Brasil). Todos os protocolos utilizados foram aplicados por via intramuscular no músculo quadríceps femoral.

Com o intuito de evitar o estresse e consequentemente a interferência nos parâmetros avaliados, os cães foram adaptados ao ambiente em que foi realizado o estudo, assim como aos avaliadores, os quais conheciam o comportamento característico dos mesmos.

Os parâmetros foram verificados e amostras sanguíneas coletadas nos tempos compreendidos de T0 a T480, sendo T0, previamente à aplicação dos fármacos e os outros tempos respeitando o intervalo de 15, 30, 60, 120, 240 e 480 minutos após a administração dos agentes.

\section{Parâmetros avaliados}

Perfil hematológico

Previamente à coleta das alíquotas sanguíneas, foi realizada tricotomia da porção ventral da região cervical respeitando-se a referência anatômica das veias jugulares externas direita e esquerda, e antissepsia com álcool $70^{\circ}$ seguido de clorexidina $0,5 \%$.

Foram coletados $2 \mathrm{~mL}$ de sangue total com seringa plástica (3 $\mathrm{mL}$ ) via punção percutânea da veia jugular direita e esquerda com agulha $(25 \times 8 \mathrm{~mm})$ distribuídas em ambos os lados, não sendo coletadas amostras de um mesmo ponto. Este material foi acondicionado em tubo contendo ácido etilenodiaminotetracético (EDTA) em quantidade suficiente para essa fração sanguínea; imediatamente os tubos foram mantidos em homogeneizador 
sanguíneo (BIOENG ${ }^{\circledR}$, São Paulo, SP, Brasil) até a realização do exame, que foi procedido em no máximo 60 minutos após cada coleta.

Os valores de eritrograma e leucograma foram obtidos com auxílio de contador automático de células (CC-530 VETERINÁRIO, CELM ${ }^{\circledR}$, Barueri, SP, Brasil) sendo o mesmo calibrado previamente ao estudo e a cada análise segundo a indicação do fabricante.

Os valores de hematócrito foram adquiridos pelo método de centrifugação (1200 rpm/5 minutos) com auxílio de centrífuga para micro-hematócrito (HB-240, CENTRIBIO ${ }^{\circledR}$, Pequim, China) calibrada anteriormente ao estudo, utilizando microcapilar com capacidade para $1 \mu \mathrm{L}$ de sangue total retirado do tubo contendo EDTA.

\section{Perfil bioquímico}

Foram coletados $3 \mathrm{~mL}$ de sangue seguindo a mesma técnica do perfil hematológico, sendo as mesmas acondicionadas em tubo sem anticoagulante com gel ativador de coágulo e colocados em rack para tubo de ensaio, mantidas em temperatura ambiente por um período de 2 horas; após, as mesmas foram centrifugadas (4000 rpm/5 minutos) com auxílio de macrocentrífuga (KC-4, Kindly ${ }^{\circledast}$, São Paulo, SP, Brasil) e separados $1,5 \mathrm{~mL}$ de soro em tubo plástico descartável com tampa e colocadas sob refrigeração $\left(2-8^{\circ} \mathrm{C}\right)$. As análises foram realizadas no prazo máximo de 12 horas após a coleta.

Os valores de albumina, AST, ALT, FA, GGT, hemoglobina, ureia e creatinina foram obtidos pelo método colorimétrico de ReitmanFrankel, utilizando espectrofotômetro (BIO-2000, BIOPLUS ${ }^{\circledast}$, São Paulo, SP, Brasil) e kit comercial específico (GoldAnalisa ${ }^{\circledR}$, Belo Horizonte, MG, Brasil) para cada variável, sendo o equipamento calibrado conforme as instruções do fabricante previamente a cada análise.

Para a determinação das proteínas totais foi utilizado o método de refratometria com auxílio de refratômetro $(\mathrm{GH}$, Optical Instrument Co., Ltd. Pequim, China) utilizando o soro sanguíneo do microcapilar, produto da separação plasmática após a centrifugação. $O$ equipamento foi calibrado com água destilada previamente as medidas.

\section{Monitoramento de outras variáveis}

Durante todo o estudo foram ainda monitoradas e registradas a frequência cardíaca (bpm) utilizando auscultação transtorácica do ciclo cardíaco por estetoscopia na região do quarto espaço intercostal esquerdo durante 1 minuto; frequência respiratória (f) avaliada através da observação dos movimentos do gradil costal do ciclo respiratório por um minuto; temperatura retal $\left({ }^{\circ} \mathrm{C}\right)$ com auxílio de termômetro clínico inserido no reto dos animais e escala de sedação de acordo com o escore de 0 a 3 , onde 0 : sem sedação; 1: sedação leve (menos alerta, mas ainda ativo); 2: sedação moderada (sonolento, mas pode deambular); e 3: sedação intensa (muito sonolento, sem capacidade de deambular).

\section{Análise estatística}

Os dados são apresentados em média e seus respectivos desvios-padrão. A análise estatística foi realizada por meio de programa computacional GraphPad PRISM v. 5 (GraphPad Software, Inc, La Jolla, CA, USA). Foi empregado o teste de normalidade de ShapiroWilk, em seguida, as amostras que apresentaram distribuição normal foram comparadas através de análise de variância (ANOVA) para amostras repetidas, seguida do teste de Bonferroni e as de distribuição anormal através do teste de Friedman. O nível de significância estabelecido foi de $5 \%$.

\section{Resultados}

Em todos os grupos o escore médio de sedação foi $2 \pm 1$, correspondendo à sedação moderada, o que permitiu a adequada coleta das amostras, verificando-se características mais proeminentes de sedação nos animais tratados com dexmedetomidina. Foi observada também redução da frequência cardíaca, respiratória e da temperatura retal em todos os momentos de avaliação. Os dados dessas variáveis estão descritos na Tabela 1.

Observou-se êmese em cinco animais do GX, um no GD e náusea em outro do GA. Notaram-se ainda tremores generalizados em dois animais do GA e relaxamento do esfíncter anal em outro deste mesmo grupo e também no GX; relaxamento da terceira pálpebra em um animal do GX.

O período entre as coletas foi adequado para o restabelecimento das variáveis analisadas uma vez que nos tempos considerados como basais, os valores dos parâmetros encontraram-se dentro da normalidade para a espécie (Kramer e Hoffman, 1997).

No tocante aos parâmetros hematológicos (Figura 1), houve redução significativa dos eritrócitos totais em todos os momentos comparados ao basal $(p<0,001)$ no $G A$, e na análise comparativa entre os grupos no tempo T480 os valores médios foram inferiores no GA em relação aos outros grupos. Foi observada redução estatisticamente significativa do hematócrito durante todos os tempos de avaliação comparativamente a T0 no grupo GA $(p<0,0001)$ e $G X(p<0,001)$. Na análise comparativa entre os grupos, os valores médios no T480 foram significativamente inferiores no GA comparados a GD $(p<0,01)$. No atinente a hemoglobina, houve redução significativa em todos os momentos comparados ao basal no GA ( $p<0,0001)$ e $G X(p<0,001)$.

Não foram verificadas alterações significativas nos valores de leucócitos comparativamente entre os grupos $(p>0,05)$. Já análise dentro de cada grupo, houve redução significativa nos momentos T30, T60, T120 e T240 no GA comparativamente ao valor basal $(p<0,05)$; nos grupos $G D$ e $G X$ os valores médios foram inferiores nos tempos T30 e T120 $(p<0,05)$, respectivamente.

Os dados referentes aos parâmetros bioquímicos estão descritos na Tabela 2. Não foram verificadas diferenças estatisticamente significativas em nenhuma das variáveis bioquímicas analisadas entre os tempos no mesmo grupo $(p>0,05)$. Na análise comparativa entre os grupos, foi verificado que os valores de albumina no tempo T0 no GX foram superiores ao GA e GD $(p<0,05)$; verificaram-se também nesta variável valores médios mais elevados no momento T120 no grupo GD comparativamente aos outros grupos $(p<0,05)$. 
Tabela 1: Frequência cardíaca (FC), respiratória $(f)$ e temperatura retal (TR) de cães submetidos a sedação com acepromazina (GA), dexmedetomidina (GD) ou xilazina (GX) em diferentes tempos de avaliação

\begin{tabular}{c|c|ccccccc}
\hline Variável & Grupo & T0 & T15 & T30 & T60 & T120 & T240 & T480 \\
\hline \multirow{2}{*}{$\begin{array}{c}\text { FC } \\
(\mathrm{bpm})\end{array}$} & GA & $140 \pm 10$ & $107 \pm 19$ & $103 \pm 28$ & $95 \pm 25$ & $115 \pm 24$ & $115 \pm 46$ & $125 \pm 16$ \\
& GD & $113 \pm 23$ & $41 \pm 12$ & $39 \pm 11$ & $39 \pm 13$ & $45 \pm 12$ & $80 \pm 21$ & $109 \pm 13$ \\
& GX & $103 \pm 19$ & $42 \pm 11$ & $45 \pm 10$ & $51 \pm 10$ & $74 \pm 10$ & $105 \pm 6$ & $124 \pm 26$ \\
\hline \multirow{2}{f}{} & GA & $22 \pm 2$ & $11 \pm 1$ & $12 \pm 2$ & $14 \pm 4$ & $13 \pm 3$ & $15 \pm 2$ & $20 \pm 7$ \\
$(\mathrm{mpm})$ & GD & $24 \pm 11$ & $12 \pm 1$ & $11 \pm 3$ & $11 \pm 3$ & $11 \pm 2$ & $17 \pm 5$ & $22 \pm 15$ \\
& GX & $25 \pm 12$ & $24 \pm 14$ & $16 \pm 9$ & $16 \pm 2$ & $12 \pm 0$ & $19 \pm 8$ & $25 \pm 4$ \\
\hline & GA & $38,9 \pm 0,4$ & $38,3 \pm 0,4$ & $38,3 \pm 0,3$ & $38 \pm 0,3$ & $37,7 \pm 0,3$ & $37,8 \pm 0,1$ & $38,2 \pm 0,1$ \\
TR & GD & $38,7 \pm 0,7$ & $38,7 \pm 0,4$ & $38 \pm 0,5$ & $37,1 \pm 0,7$ & $36,1 \pm 1,1$ & $37,7 \pm 0,7$ & $38,5 \pm 0,3$ \\
& GX & $38,8 \pm 0,1$ & $38,1 \pm 0,1$ & $37,6 \pm 0,4$ & $36,8 \pm 0,2$ & $36,9 \pm 0,7$ & $38,4 \pm 0,3$ & $38,8 \pm 0,4$ \\
\hline
\end{tabular}

Tabela 2: Média \pm desvio-padrão da albumina, ALT, AST, FA, GGT, creatinina e ureia em cães submetidos a sedação com acepromazina (GA), dexmedetomidina (GD) ou xilazina (GX) em diferentes tempos de avaliação.

\begin{tabular}{|c|c|c|c|c|c|c|c|c|}
\hline Variável & Grupo & TO & T15 & T30 & $\mathrm{T} 60$ & $\mathrm{~T} 120$ & T240 & T480 \\
\hline \multirow{3}{*}{$\begin{array}{l}\text { Albumina } \\
\text { (g/dL) }\end{array}$} & $\mathrm{GA}$ & $3,9 \pm 1,1$ & $3,4 \pm 0,8$ & $3,7 \pm 1,2$ & $3,4 \pm 0,9$ & $4,7 \pm 1^{*}$ & $3,3 \pm 0,8$ & $3,3 \pm 1,1$ \\
\hline & GD & $2,1 \pm 0,8$ & $2,4 \pm 0,7$ & $2 \pm 0,9$ & $2,5 \pm 1,5$ & $2 \pm 0,4$ & $2,6 \pm 1,2$ & $2,3 \pm 0,8$ \\
\hline & GX & $4,5 \pm 1,5^{\star}$ & $4 \pm 1,8$ & $3,9 \pm 1,2$ & $3,9 \pm 0,9$ & $4,2 \pm 1$ & $4,3 \pm 0,8$ & $3,9 \pm 1,1$ \\
\hline \multirow{3}{*}{$\begin{array}{c}\text { ALT } \\
(\mathrm{UI} / \mathrm{L})\end{array}$} & GA & $26,3 \pm 12,8$ & $24,8 \pm 12,2$ & $22,6 \pm 11$ & $24,8 \pm 11,9$ & $24,2 \pm 11,7$ & $24 \pm 14,3$ & $25,1 \pm 12,4$ \\
\hline & GD & $25,7 \pm 13,6$ & $28 \pm 13,9$ & $30,8 \pm 17,3$ & $31,3 \pm 16,4$ & $34 \pm 19,2$ & $37,3 \pm 23,6$ & $34,3 \pm 22,7$ \\
\hline & GX & $33,3 \pm 18,9$ & $33,5 \pm 21,7$ & $40,4 \pm 21,5$ & $32,6 \pm 20,2$ & $37,8 \pm 14$ & $36,5 \pm 18,6$ & $36,7 \pm 20,5$ \\
\hline \multirow{3}{*}{$\begin{array}{c}\text { AST } \\
(\mathrm{UI} / \mathrm{L})\end{array}$} & GA & $42,4 \pm 16,2$ & $38,6 \pm 13,4$ & $42,4 \pm 11,7$ & $43,1 \pm 11,8$ & $46 \pm 11,9$ & $47,4 \pm 12,6$ & $49 \pm 10,8$ \\
\hline & GD & $53,2 \pm 19,4$ & $53,5 \pm 18,4$ & $46,7 \pm 17,6$ & $58 \pm 23,9$ & $51,7 \pm 19,7$ & $58,8 \pm 15,5$ & $54,5 \pm 13,5$ \\
\hline & GX & $55,8 \pm 14,7$ & $49,6 \pm 12,8$ & $49,7 \pm 13,1$ & $47,8 \pm 12$ & $50,9 \pm 10,2$ & $56,3 \pm 19,2$ & $59,7 \pm 18,8$ \\
\hline \multirow{3}{*}{$\begin{array}{c}\text { FA } \\
(\mathrm{UI} / \mathrm{L})\end{array}$} & $\mathrm{GA}$ & $64 \pm 28$ & $43 \pm 25$ & $63 \pm 31$ & $59 \pm 22$ & $54 \pm 17$ & $58 \pm 21$ & $53 \pm 21$ \\
\hline & GD & $52 \pm 16$ & $56 \pm 21$ & $49 \pm 18$ & $53 \pm 17$ & $51 \pm 18$ & $54 \pm 17$ & $54 \pm 18$ \\
\hline & GX & $60 \pm 28$ & $48 \pm 15$ & $44 \pm 10$ & $52 \pm 26$ & $40 \pm 16$ & $56 \pm 14$ & $57 \pm 17$ \\
\hline \multirow{3}{*}{$\begin{array}{c}\text { GGT } \\
(\mathrm{UI} / \mathrm{L})\end{array}$} & GA & $4,6 \pm 2,4$ & $2,8 \pm 2,1$ & $2,2 \pm 1,6$ & $3,8 \pm 1,6$ & $3,4 \pm 1,6$ & $3,4 \pm 2,3$ & $4,4 \pm 1,4$ \\
\hline & GD & $2,6 \pm 1,4$ & $3,3 \pm 1,4$ & $2,7 \pm 1,2$ & $3,3 \pm 1,4$ & $2,3 \pm 1,6$ & $2,9 \pm 1,7$ & $2,9 \pm 1,7$ \\
\hline & GX & $1,5 \pm 2$ & $3,3 \pm 2,2$ & $2,8 \pm 2,5$ & $2,8 \pm 2,2$ & $2,3 \pm 2,3$ & $3 \pm 2$ & $1,8 \pm 1,8$ \\
\hline \multirow{3}{*}{$\begin{array}{c}\text { Creatinina } \\
(\mathrm{mg} / \mathrm{dL})\end{array}$} & GA & $0,8 \pm 0,1$ & $0,8 \pm 0,1$ & $0,8 \pm 0,1$ & $0,8 \pm 0,2$ & $0,8 \pm 0,1$ & $0,8 \pm 0,2$ & $0,8 \pm 0,1$ \\
\hline & GD & $0,7 \pm 0,1$ & $0,7 \pm 0,1$ & $0,7 \pm 0,1$ & $0,7 \pm 0,1$ & $0,7 \pm 0,1$ & $0,7 \pm 0,1$ & $0,7 \pm 0,1$ \\
\hline & GX & $0,9 \pm 0,2$ & $0,9 \pm 0,2$ & $0,9 \pm 0,2$ & $0,9 \pm 0,2$ & $1 \pm 0,2$ & $1 \pm 0,2$ & $1 \pm 0,4$ \\
\hline \multirow{3}{*}{$\begin{array}{l}\text { Ureia } \\
\text { (mg/dL) }\end{array}$} & GA & $33,2 \pm 11,5$ & $33,8 \pm 12,8$ & $33,6 \pm 11,7$ & $31,6 \pm 7,2$ & $33 \pm 9,1$ & $38 \pm 15,6$ & $31,8 \pm 7,2$ \\
\hline & GD & $32,5 \pm 9,8$ & $31,7 \pm 9,2$ & $32,8 \pm 10,6$ & $32,2 \pm 9,3$ & $32 \pm 8$ & $29,3 \pm 5,9$ & $29,2 \pm 5,7$ \\
\hline & GX & $35,2 \pm 12,1$ & $34,2 \pm 10,7$ & $34,2 \pm 6,2$ & $34,8 \pm 8,3$ & $44,2 \pm 6,4$ & $40,7 \pm 8,8$ & $39,5 \pm 13,6$ \\
\hline
\end{tabular}

* Diferença entre os grupos $(p<0,05)$ 

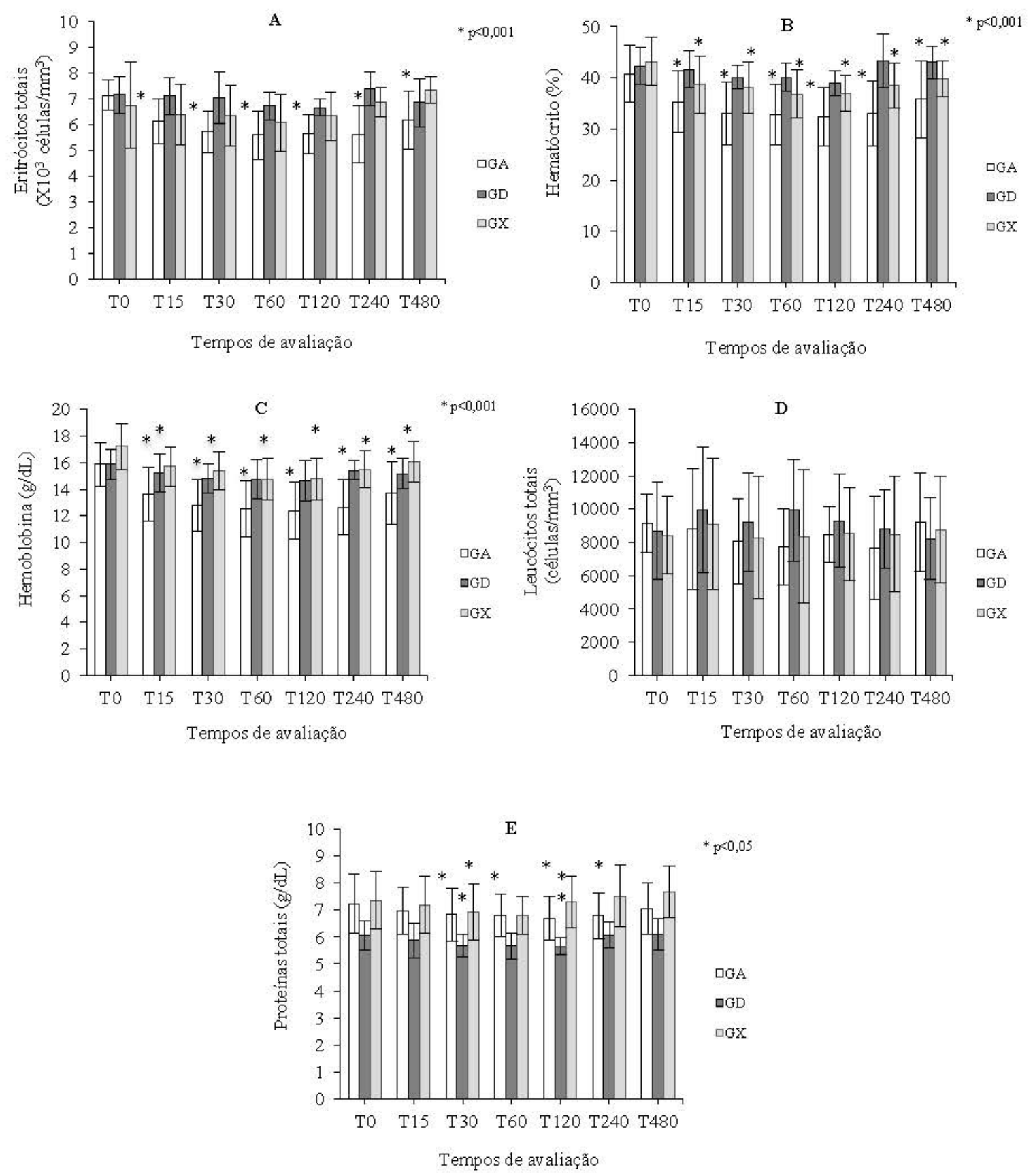

Figura 1: Variação dos valores médios \pm desvio-padrão dos eritrócitos totais $(A)$, hematócrito $(B)$, hemoglobina (C), leucócitos totais (D) e proteínas totais (E) cães submetidos a sedação com acepromazina (GA), dexmedetomidina (GD) ou xilazina (GX) em diferentes tempos de avaliação. * Diferença em relação a T0

\section{Discussão}

As doses dos fármacos empregadas no presente estudo são aquelas descritas e utilizadas na rotina clínica para cães (Cortopassi e Fantoni, 2010; Kuusela et al., 2000), e a escolha dos tempos de coleta foram baseadas nos estudos farmacocinéticos dos respectivos agentes (Garcia-Villar et al. 1981; Hashem et al., 1992; Kuusela et al., 2000), que embora estes autores tenham mostrado em suas pesquisas níveis plasmáticos das referidas substâncias em tempos superiores aos utilizados, optamos por reduzir os tempos coleta em função da possível hipovolemia, influenciando diretamente nos resultados.
Alterações nos parâmetros hematológicos podem ser ocasionadas por diversos mecanismos, dentre eles, estresse, marginalização celular e sequestro por alguns órgãos (Wilson et al., 2004).

Além dos efeitos centrais que determinam as características sedativas ou tranquilizantes dos fenotiazínicos e agonistas alfa 2-adrenérgicos, essas substâncias também podem atuar perifericamente, mais especificamente sobre os receptores alfa 1-adrenérgicos pós-sinápticos, podendo, desta forma, influenciar diretamente nos parâmetros hematológicos devido à ocorrência de migração de células sanguíneas para periferia 
(Farver et al.,1986; Kullmann et al., 2011). No presente estudo, foi possível verificar esse efeito, principalmente nos animais tratados com acepromazina e xilazina, em que os valores reduziram em até $20 \%$.

O estudo clássico avaliando os efeitos da acepromazina na dose de $0,07 \mathrm{mg} / \mathrm{kg}$ por via intramuscular em cães foi realizado por Lang et al. (1979) onde observaram redução do hematócrito em $23 \%$, sendo que a maior redução ocorreu aos 60 minutos da administração do agente. Segundo os autores, tal redução ocorre devido à supressão adrenérgica, levando a um aumento esplênico e consequentemente sequestro dessas células. Wilson et al. (2004) compararam a redução no valor do hematócrito com a esplenomegalia em cães que receberam a acepromazina $(0,044 \mathrm{mg} / \mathrm{kg})$ como parte de quatro protocolos anestésicos, e verificaram redução dos valores de hematócrito quando comparados ao valor basal, porém não observaram correlação entre a área esplênica e a redução dessa variável e, alegaram que o sequestro de hemácias provavelmente ocorre em outros órgãos como fígado, pele e músculos e não apenas no baço.

Habib et al. (2002) utilizando xilazina $(0,18 \mathrm{mg} / \mathrm{kg})$ ou clorpromazina $(8 \mathrm{mg} / \mathrm{kg}$ ) em ovinos, administrados por via intramuscular observaram redução significativa nos valores de hematócrito e hemoglobina, e atribuíram esse achado aos efeitos periféricos dos fármacos. Kiliç (2008) avaliou alterações hematológicas e bioquímicas em seu estudo com bovinos utilizando a associação de detomidina, midazolam e cetamina por via intravenosa, e observou redução nos valores de eritrócitos, hematócrito e hemoglobina, alegando que tal efeito tenha ocorrido principalmente devido à redução da atividade simpática causada no baço e outros reservatórios de células sanguíneas, mediante o emprego da detomidina, além do fato da possível hemodiluição pelo deslocamento do fluido extravascular para o meio intravascular.

De maneira semelhante Kullmann et al. (2011) verificaram que em equinos tratados com detomidina ou xilazina há redução do hematócrito e, segundo esses autores, os principais mecanismos envolvidos na redução dessa variável são a lise eritrocitária, saída de fluidos do meio extravascular para o intravascular na tentativa de manutenção do débito cardíaco e o sequestro esplênico.

A dexmedetomidina é um composto imidazólico derivado da medetomidina, sendo a estrutura química constituída do seu isômero dextrógero determinando alta seletividade a alfa 2 adrenorreceptores (relação alfa2:alfa1 de 1620:1). Com isso, possui efeitos sedativos mais potentes sem a importante depressão cardiovascular (Jorden; Tung, 2002). Assim sendo, pode-se supor que essa maior seletividade pode determinar também menor marginalização celular, corroborando desta forma com os estudos encontrados no estudo em tela.

Os dados apresentados no presente estudo demonstraram que os três protocolos empregados não causaram maiores alterações sobre as variáveis bioquímicos hepáticas analisadas, não caracterizando portanto, hepatotoxicidade.

Os fármacos ou seus metabólitos podem promover alterações mitocondriais nos hepatócitos e hepatotoxicidade por diversos mecanismos, sendo eles: 1 ) reações relacionadas com enzimas do citocromo P450 comprometendo a homeostase do hepatócito e, consequentemente, sua ruptura; 2) disfunção de proteínas transportadoras relacionadas com o fluxo de ácidos biliares; 3) reações imunes aos metabólitos formados no fígado; 4) hepatotoxicidade promovida por células inflamatórias; 5 ) apoptose mediada por TNF e 6) estresse oxidativo (Jaeschke et al., 2002; Labbe et al., 2008), tendo como destaque o primeiro mecanismo, visto a ligação direta com os processos de biotransformação dos fármacos, principalmente ligados às reações de fases I e II (Lee 2003).

Hepatotoxicidade mediada por fármacos tranquilizantes ou anestésicos, e baseada em dosagens enzimáticas já foram objeto de estudo em outras pesquisas e mostraram boa relação na detecção de alterações agudas ou crônicas induzidas por esses agentes (Topal et al., 2003; Turillazzi et al., 2007; Dima, 2009; Mattos Junior et al., 2009).

A biotransformação dos compostos fenotiazínicos em humanos é mediada por inúmeras enzimas derivadas dos hepatócitos e envolve reações de fases I e II, formando, consequentemente, metabólitos que são eliminados pelas fezes e urina (Neves, 2003). Em equinos, a biotransformação da acepromazina, um fármaco deste grupo, ocorre de maneira diferente, sendo que a maioria dos metabólitos não são conjugados nesta espécie e são eliminados quase que de forma inalterada pelos rins (Dewey et al., 1981).

Os agonistas alfa 2-adrenérgicos também passam pelo processo de biotransformação no fígado por meio de enzima do sistema citocromo P450, e também por glicuronização, não ocorrendo a formação de metabólitos ativos ou tóxicos para pacientes hígidos (Panzer et al., 2011).

Não foi objetivo do presente estudo verificar os efeitos dos metabólitos dos fármacos que foram objeto da pesquisa, porém, baseado nas informações geradas pelos autores supracitados, fica evidente que essas substâncias não são potencialmente tóxicas para os animais que apresentem tais vias metabólicas intactas. Desta forma, acredita-se que tal situação tenha ocorrido no estudo em tela, pois conforme mencionado, nenhuma das provas bioquímicas apresentou alterações durante o período avaliado. Ademais, alguns metabólitos podem ser excretados de maneira intacta, o que em condições fisiológicas não causaria nenhum comprometimento aos hepatócitos (Zimmerman, 1999).

Pacientes em condições de anemia, hipovolemia ou com hepatopatias de diferentes formas podem apresentar importantes alterações na oxigenação tecidual ou inabilidade em biotransformar os fármacos administrados (Muir, 2007). Animais nestas condições podem apresentar descompensação hemodinâmica na iminência da utilização da acepromazina ou xilazina, visto que os resultados desta pesquisa, em pacientes hígidos, apontam importante redução dos valores de eritrócitos, hematócrito e hemoglobina. Já a administração da dexmedetomidina pode ser utilizada com relativa segurança nestas condições. A administração de uma única dose de todos os fármacos não induziu alterações sobre a função hepática, porém sugerem-se novos estudos com os respectivos agentes por períodos mais longos de avaliação com o objetivo de elucidar os danos hepáticos dessas substâncias a nível crônico.

\section{Conclusões}

Baseado nos resultados desta pesquisa e nestas condições experimentais, pode concluir que:

- A acepromazina na dose empregada promove redução dos valores de eritrócitos totais, hematócrito e hemoglobina por até 8 horas da aplicação; 
- A xilazina promove redução dos valores do hematócrito e hemoglobina pelo mesmo período;

- A dexmedetomidina não promoveu alterações em nenhum dos parâmetros hematológicos avaliados durante 8 horas;

\section{Referências}

AMBRISKO, T.D.; HIKASA, Y. Neurohormonal and metabolic effects of medetomidine compared with xylazine in beagle dogs. Canadian Journal Veterinary Research, v. 66, n. 1, p. 42- 49, 2002. DEWEY, E.A.; MAYLIN, G.A.; EBEL, J.G.; HENION, J.D. The metabolism of promazine and acetylpromazine in the horse. Drug metabolism and disposition, v. 9, n. 1, p. 30-36, 1981.

DIMA, L. Pharmacokinetic interactions of new antipsychotics with other psychotropic drugs. Bulletin Transilvania University of Braşov, v. 2, n. 51, p. 31-38, 2009.

CORTOPASSI, S.R.G.; FANTONI, D.T. Medicação pré-anestésica. In: FANTONI, D.T.; CORTOPASSI, S.R.G. Anestesia em cães e gatos. 2. ed. São Paulo: Roca, 2010. p. 217-227.

FARVER, T.B.; HASKINS, S.C.; PATZ, J.D. Cardiopulmonary effects of acepromazine and of the subsequent administration of ketamine in the dog. American Journal Veterinary Research, v. 47, n. 3, p. 631-635, 1986

GARCIA-VILLAR, R.;TOUTAIN, P.L.;ALVINERIE, M. RUCKEBUSH, Y.J. The pharmacokinetics of xylazine hydrochloride: an interspecific study. Journal Veterinary Pharmacology Therapeutics, v. 4, n. 2, p. 87-92, 1981

HABIB, S.; DAS, B.C.; ISLAM, M.N.; HOSSAIN, M.K.; AHMED, M.F. A comparison of xylazine, diazepam, chlorpromazine and prometazine in relation to certain clinical and hematological parameters of indigenous sheep (Ovis aries). Pakistan Journal Biological Sciences, v. 5, n. 4, p. 484-488, 2002.

HASHEM, A.; KIETZMANN, M.; SCHERKL, R. The pharmacokinetics and bioavailability of acepromazine in the plasma of dogs. Deutsche Tierarztliche Wochenschrift, v. 99, n. 10, p. 396-398, 1992.

JAESCHKE, H.; GORES, G.J.; CEDERBAUM, A.L.; HINSON, J.A.; PESSAYRE, D.; LEMASTERS, J.J. Mechanisms of hepatotoxicity. Toxicological Sciences, v. 65, n. 2, p. 166-176, 2002.

JORDEN, V.S.B.; TUNG, A. Dexmedetomidine: clinical update Seminars Anesthesia, Perioperative Medicine Pain, v. 21, n. 4, p. 265-274, 2002.

KRAMER, J.W.; HOFFMAN, W. Clinical enzymology. In: KANEKO, J.J.; HARVEY, J.W.; BRUSS, M.L. Clinical Biochemistry Domestic Animals, 5. th ed. San Diego: Academic Press, 1997. p. 303-323.

KILIÇ, N. Cardiopulmonary, biochemical and haematological changes after detomidine-midazolam-ketamine anaesthesia in calves. Bulletin Veterinary Institute Pulawy, v. 52, n. 3, p. 453-456, 2008.

KOJIMA, K.; NISHIMURA, R.; MUTOH, T.; HONG, S.; MOCHIZUKI, M.; SASAKI, N. Effects of medetomidine-midazolam, acepromazinebutorphanol, and midazolam-butorphanol on induction dose of thiopental and propofol and on cardiopulmonary changes in dogs. American Journal Veterinary Research, v. 63, n. 12, p. 1671-1679, 2002.

KRAMER, J.W.; HOFFMAN, W. Clinical enzymology. In: KANEKO, J.J.; HARVEY, J.W.; BRUSS, M.L. Clinical Biochemistry in Domestic Animals, 5. th ed. San Diego: Academic Press, 1997. p. 303-323.

KULLMANN, A.; SANZ, M.; FOSGATE, G.; SAULEZ, M.; PAGE, P.; RIOJA, E. Effects of xylazine, romifidine and detomidine on haematology, biochemistry and splenic size in horses. Journal Veterinary Emergency Critical Care, v. 21, suppl. 1, p. 16, 2011.
- Nenhum dos fármacos empregados promoveu alterações sobre a função hepática e renal avaliadas por meio das dosagens enzimáticas, creatinina e ureia por até 8 horas de avaliação.

KUUSELA, E.; RAEKALLIO, M.; ANTITILA, M.; FALCK, I.; MOLSA, S.; VAINIO, O. Clinical effects and pharmacokinetics of medetomidine and its enantiomers in dogs. Journal Veterinary Pharmacology Therapeutics, v. 23, n. 1, p. 15-20, 2000.

LABBE, G.; PESSAYRE, D.; FROMENTY, B. Drug-induced liver injury through mitochondrial dysfunction: mechanisms and detection during preclinical safety studies. Fundamental Clinical Pharmacology, v. 22, n. 4, p. 335-353, 2008.

LANEVSCHI, A.; WARDROP, K.J. Principles of transfusion medicine in small animals. Canadian Veterinary Journal, v. 42, n. 6, p. 447-454, 2001.

LANG, S.M., EGLEN, R.M., HENRY, A.C. Acetylpromazine administration: its effect on canine hematology. Veterinary Record, v. 105 , n. 17 , p. $397-398,1979$

LEE, W.M. Drug-induced hepatotoxicity. New England Journal Medicine, v. 349, n. 5, p. 474-485, 2003.

MATTOS JUNIOR, E.; RUSSO, C.; SANTOS, G.J.V.G.; BALARIN, M.R.S.; SAUT, J.P.E. Avaliação da função hepática em cães submetidos a anestesia pela associação zolazepam/tiletamina. Semina: Ciências Agrárias, v. 30, n. 2, p. 417-424, 2009.

MUIR, W.W. Considerations for general anesthesia. In: TRANQUILLI, W.J.; THURMON, J.C.; GRIMM, K.A. Lumb \& Jones' veterinary anesthesia and analgesia. 4. th. ed. 2007. p. 7-30.

NEVES, E.T.A.; MARGONATO, E.B.; NISHIYAMA, P., BANDO, E.; MACHINSKI Jr., M. Avaliação do ensaio imediato para a pesquisa de compostos fenotiazínicos na urina utilizando o reagente de forrest modificado. Revista Brasileira Toxicologia, v. 16, n. 1, p. 122, suppl, 2003.

PANZER, O.; MOITRA, V.; SLADEN, R.N. Pharmacology of sedative-analgesic agents: dexmedetomidine, remifentanil, ketamine, volatile anesthetics, and the role of peripheral $\mathrm{Mu}$ antagonists. Anesthesiology Clinics, v. 29, n. 4, p. 587-605, 2011.

THURMON, J.C.; TRANQUILLI, W.J.; BENSON, G.J. Preanesthetics and anesthetic adjunts. In: Lumb and Jones veterinary anaesthesia. 3th ed. Baltimore: William and Wilkins, 1996, p.183-209.

TOCCI, L.J. Transfusion medicine in small animal practice. Veterinary Clinical North America Small Animal Practice, v. 40, n. 3, p. 485-494, 2010.

TOPAL, A.; GÜL, N.; ILÇÖL, Y.; GÖRGÜL, O.S. Hepatic effects of halothane, isoflurane or sevoflurane anesthesia in dogs. Journal Veterinary Medical Association Physiology, Pahtology, Clinical Medicine, v. 50, n. 10, p. 530-533, 2003.

TURILLAZZI, E.; D'ERRICO, S.; NERI, M.; RIEZZO, I.; FINESCHI, $\mathrm{V}$. A fatal case of fulminant hepatic necrosis following sevoflurane anesthesia. Toxicologic Pathology, v. 35, n. 6, p. 840-845, 2007.

WILSON, D.V.; EVANS, A.T.; CARPENTER, R.E.; MULLINEAUX, D.R. The effect of four anesthetic protocols on splenic size in dogs. Veterinary Anaesthesia Analgesia, v. 31, n. 2, p. 102-108, 2004.

ZIMMERMAN, H.J. Vulnerability of the liver to toxic injury. In: ZIMMERMAN, H.J. Hepatotoxicity, 2. ed. Philadelphia: Lippincott Williams \& Wilkins, 1999. p. 41-60. 